\title{
Anode-Shielded, Sputter-Deposited Nanocrystalline Sn Thin-Film Anodes for Lithium-Ion Batteries
}

\author{
K.-F. Chiu, ${ }^{\mathrm{a}, *, \mathrm{z}}$ H. C. Lin, ${ }^{\mathrm{b}}$ K. M. Lin, ${ }^{\mathrm{a}}$ T. Y. Lin, ${ }^{\mathrm{a}}$ and D. T. Shieh ${ }^{\mathrm{c}, *}$ \\ ${ }^{a}$ Department of Materials Science and Engineering, Feng Chia University, \\ 407 Taichung, Taiwan \\ ${ }^{b}$ Department of Materials Science and Engineering, National Taiwan University Taiperi, Taiwan \\ ${ }^{c}$ Industrial Technology Research Institute, Materials Research Laboratories, Hsinchu, Taiwan
}

\begin{abstract}
Sn thin-film anodes have been prepared using radio frequency driven magnetron sputtering. With appropriate anode shielding on the sputter gun during deposition, film crystallinity and surface morphology can be well controlled. The anode shielding changed the plasma ion density, resulting in lower ion flux available on the substrate. Sn thin-film anodes deposited with anode shielding can develop fine and smooth morphology composed of ultrasmall particles $(5-10 \mathrm{~nm})$ uniformly dispersed on the surface. Grazing-angle X-ray diffraction and field-emission scanning electron microscopy were used to characterize the film crystallinity and surface morphology. The electrochemical properties of the thin-film anodes deposited under various conditions were measured and compared. High reversible capacity with low first-cycle capacity loss can be obtained. During the first 15-20 cycles significant capacity loss was observed, but at the next cycles capacity became more stable. The cycling properties of the Sn thin-film anodes were improved significantly.

(C) 2006 The Electrochemical Society. [DOI: 10.1149/1.2184923] All rights reserved.
\end{abstract}

Manuscript submitted August 30, 2005; revised manuscript received January 13, 2006. Available electronically March $28,2006$.

Lithium-ion secondary batteries have recently received extensive attention for applications in portable power sources, particularly for lightweight, compact $3 \mathrm{C}$ electronics, due to their high and stable working voltage, superior energy density, low self-discharge rate, and long cycling life. Alloy-type anodes are attractive anode materials due to high-capacity storage, which is twice as high as commercial carbonaceous anodes. They have been considered as alternative options for many years. ${ }^{1-3}$ Recently, Sn anodes as a typical example have been reviewed and investigated in detail. ${ }^{4-6}$ However, the main drawback of this system is the drastic volume change due to lithium insertion/removal to/from host materials during charge/ discharge cycles. The volume change often results in huge mechanical stress, which leads to poor electrochemical performance. For Sn anodes, it has been reported that minimizing the Sn particle sizes can effectively improve the cycling performances. ${ }^{1}$ Reducing the mobility of Sn atoms can prevent agglomeration of large clusters during sample preparation and charge-discharge processes and enhance the cell performance. Therefore, some researchers have shown that alloying with other metals to form nanocrystalline/ amorphous structure was an effective option to enhance the cycling ability. ${ }^{1,7-9}$ However, as a relative soft material with low melting temperature, the elemental Sn can readily form large clusters during fabrication. Most of the published results reveal the coarse and wellcrystallized nature of elemental Sn. ${ }^{1-8}$ There is little literature on the preparation and characterization of nanocrystalline/amorphous elemental Sn electrodes.

In this study, Sn thin films were deposited by an anode-shielded sputtering technique. Thin-film Sn anodes with nanosized surface features (particles) have been fabricated. The sizes of surface particles/grains can be well tuned. The electrochemical properties of the deposited films were evaluated by half-cell charge-discharge tests. The cycling performance of the nanocrystalline $\mathrm{Sn}$ anodes has shown significant improvement.

\section{Experimental}

The Sn thin films were prepared by radio frequency (rf) driven magnetron sputtering and deposited on p-type $\mathrm{Si}(100)$ wafers or stainless steel substrates using a pure Sn (99.99\%) target. Figure 1 shows the setup of the sputter system. An additional anode shielding was equipped on the target, which can modulate the properties of plasma. The ion current measured on the substrate holder showed that the anode shielding can confine the plasma and therefore reduce

\footnotetext{
* Electrochemical Society Active Member.

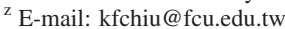

the deposition flux of ions which bombard the growing films. Because for magnetron sputtering most of the sputtered atoms remain neutral, the bombarding ions are Ar ions from the plasma gas. Ion bombardment transfers the energy and momentum of the Ar ions to the adatoms, and therefore influencing the film properties. Films deposited with/without anode shielding (labeled AS and n-AS) have been studied and $\mathrm{Sn}$ films were also deposited with/without anode shielding under different substrate bias (labeled AS, AS10V $A S 30 V_{D C}, \quad A S 50 V_{D C}, A S 70 V_{D C}$, and n-AS, n-AS10V $V_{D C}$, n$A S 30 V_{D C}, n-A S 50 V_{D C}$, respectively). The substrate holder was grounded when it was not biased. The diameter of the target was 2 in. The anode shielding was made of stainless steel and was grounded during deposition. The rf gun power was $40 \mathrm{~W}$. The distance from target to substrate was $8.5 \mathrm{~cm}$. The working gas for deposition was $99.99 \%$ pure Ar and the gas flow was $15 \mathrm{sccm}$ during sputtering. The working pressure was maintained at $1.33 \mathrm{~Pa}$ for all deposition runs. The substrate temperature was monitored and was less than $50^{\circ} \mathrm{C}$ for $\mathrm{AS}, \mathrm{n}-\mathrm{AS}$, and biased processes after $20 \mathrm{~min}$
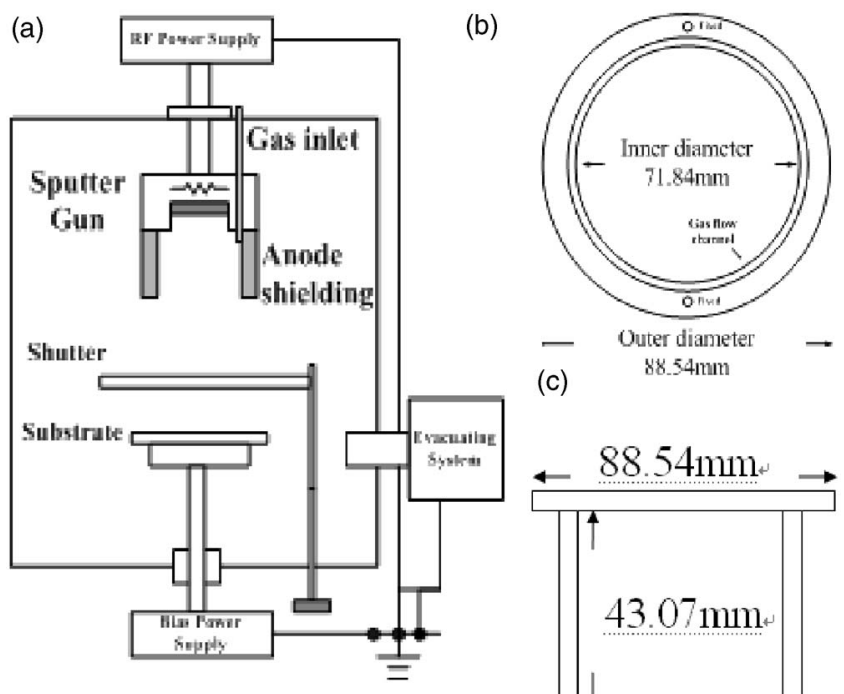

(c)

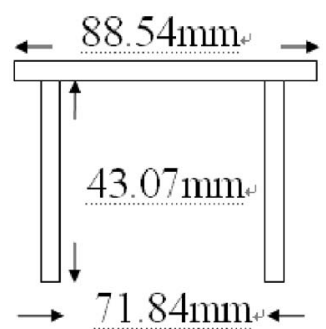

Figure 1. (a) Schematic diagram of anode shielding-assisted magnetron sputter system and schematic diagram of anode shielding, (b) top view, and (c) cross-sectional view. 


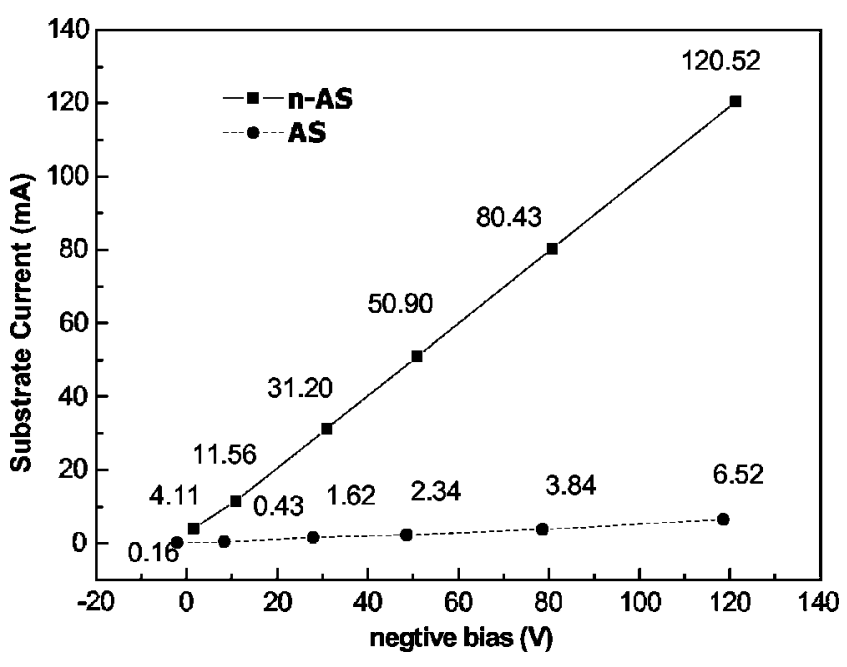

Figure 2. Current measured on the substrate holder with different bias during the deposition of n-AS and AS films.

of deposition. It was therefore a low-temperature deposition process. By controlling proper deposition times, Sn films prepared here have the same thickness of $\sim 120 \mathrm{~nm}$, which was measured by an $\alpha$-step profilometer (Veeco Instrument, Inc., Dektak 3030ST). The deposition times needed are summarized in Table I. The bias-induced ion bombardment can provide energy to assist chemisorption and incorporation of adatoms, whereas to sever ion bombardment causes resputtering (etching). Therefore, deposition rates change with applied bias. The mass of the Sn films was obtained by multiplying the mass deposition rate $\left(\mathrm{mg} / \mathrm{s} \mathrm{cm}^{2}\right)$ with deposition time. Ideal density was assumed for the measurement of mass deposition rates. However, because the actual film density varies with deposition conditions, the capacities $(\mathrm{mAh} / \mathrm{g})$ are therefore subject to an uncertainty of $\pm 5 \%$.

Because the deposited films are relatively thin $(\sim 120 \mathrm{~nm})$, a normal powder X-ray diffractometer is not able to provide appreciable information. The film crystallography was characterized by grazing-angle X-ray diffraction (XRD) using a Rigaku D/MAX2500 $\mathrm{X}$-ray diffractometer with $\mathrm{Cu} \mathrm{K} \alpha$ radiation (wavelength $1.5405 \AA$ ). The resulting $\mathrm{S} / \mathrm{N}$ ratios for grazing-angle XRD are satisfactory. Some results showing lower $\mathrm{S} / \mathrm{N}$ ratios are due to the amorphous features of deposited films rather than the measurements. The surface morphology was observed by a JOEL JSM-6700 field-emission scanning electron microscope (FESEM). The charge-discharge and cycle life tests were performed using Autolab PGSTAT30 and Jeihan 5000 potentiostat/galvanostat systems. Half-cells made of the films prepared under different conditions were measured. Lithium metal was used as counter and reference electrodes, and the electrolyte was $1 \mathrm{M} \mathrm{LiPF}_{6}$ and ethylene carbonate/ethyl methyl carbonate (volume ratio 1:2) solution. Low-rate operation can causes anomalous, high-voltage irreversible capacity due to the decomposition of electrolyte. To avoid this phenomenon, a rapid charge scheme to below $0.8 \mathrm{~V}$ has been suggested. ${ }^{5}$ Therefore, the half-cells were charged (lithiated) and discharged (delithiated) at 50 and $10 \mu \mathrm{A} \mathrm{cm}^{-2}$ (current per unit surface of stainless steel substrate) at a constant temperature of $25^{\circ} \mathrm{C}$. Typically for 120 -nm-thick Sn films, the first discharge took $\sim 2 \mathrm{~h}$. All cells were assembled and tested in an Arfilled glove box.
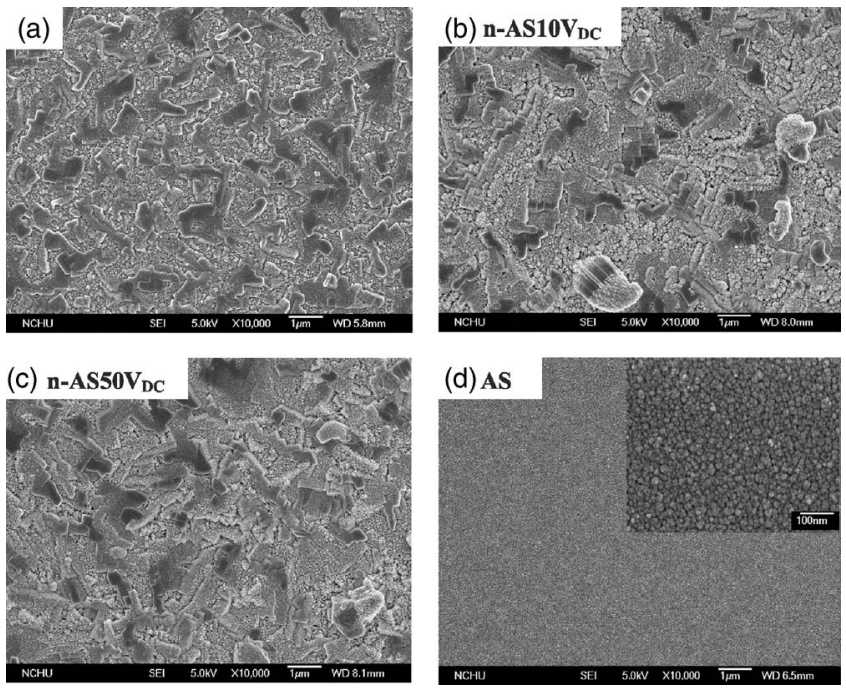

Figure 3. Surface morphology of n-AS Sn thin films deposited under bias of (a) 0 , (b) 10, and (c) $50 \mathrm{~V}$. The AS Sn thin film at the same magnification is shown in (d) for comparison.

\section{Results and Discussion}

Process diagnostics. - Figure 2 shows the currents measured on the copper substrate holder as a function of different negative substrate bias provided with/without anode shielding (AS and n-AS) during sputtering. It is obvious that the currents with anode shielding are much smaller than those without anode shielding. The much different plasma characteristics with and without anode shielding indicate the amounts of ion flux (Ar ions in this case) irradiating on the growing films of the substrate holder are different. During the deposition, the anode shielding can be viewed as a grounded wall surrounding the plasma, which effectively confines the plasma in a much smaller volume compared with the vacuum chamber in the case of n-AS. Only a small portion of plasma ions can reach the substrates during deposition, which corresponds to the smaller currents as shown in Fig. 2. In magnetron sputtering, most sputtered atoms remain neutral; therefore, the measured positive currents are composed of $\mathrm{Ar}$ ions from the plasma gas, and the electrons are repelled by the negative bias. For deposition without anodeshielding (n-AS), electrons in plasma easily spread to a large volume and reach the chamber wall and the substrate, as the magnetron gun in our system is not perfectly balanced. Since the plasma bulk should maintain neutral, the positive Ar ions also follow the electrons. The substrates are therefore immersed in the plasma and subject to larger flux of ion bombardment. Consequently, a much larger ion flux is available in the n-AS case. These ions bombard on the film surface with energy equal to the potential difference between the plasma and substrates. It provides extra energy to the growing films and may influence the film crystallography and morphology. The influences of the extra energy provided by ion bombardment are even more significant for low-melting metals or alloys, ${ }^{10}$ which may result in well-crystallized Sn films. In addition, we suppose that for the anode-shielded films (AS) there is lower ion flux, as measured, and therefore less integral energy for grain growth, resulting in the nanosized surface morphology as shown in the following sections.

Table I. Deposition times needed for 120-nm Sn thin films deposited under different conditions.

\begin{tabular}{cccccc} 
Samples & $0 \mathrm{~V}$ & $10 \mathrm{~V}$ & $30 \mathrm{~V}$ & $50 \mathrm{~V}$ & $70 \mathrm{~V}$ \\
\hline $\mathrm{AS}$ & $19 \mathrm{~min}, 20 \mathrm{~s}$ & $19 \mathrm{~min}, 20 \mathrm{~s}$ & $20 \mathrm{~min}$ & $21 \mathrm{~min}, 30 \mathrm{~s}$ & $14 \mathrm{~min}$
\end{tabular}



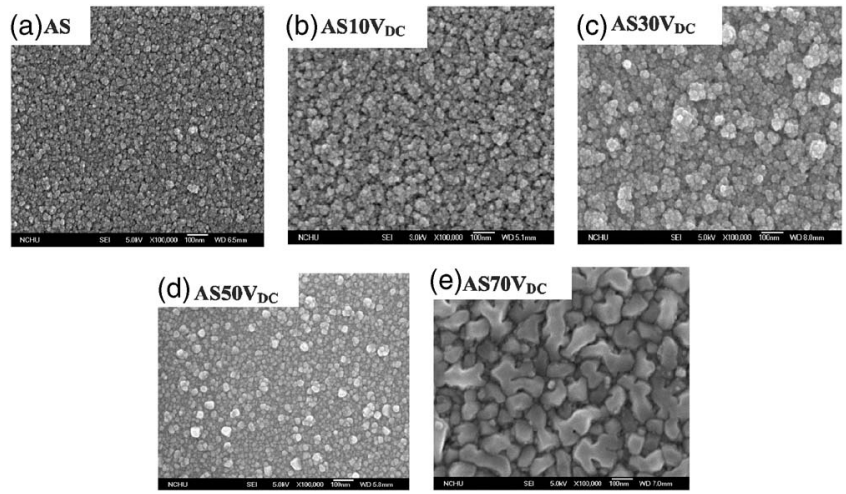

Figure 4. Surface morphology of AS Sn thin films deposited under different bias of (a) 0, (b) 10, (c) 30, (d) 50, and (e) $70 \mathrm{~V}$.

Surface morphology.- Figure 3a-c shows the surface morphologies of n-AS samples. The surface morphologies of n-AS Sn films are coarse and rough. Large particles with the size around $1 \mu \mathrm{m}$ randomly disperse on the film surface. Similar results are commonly observed for sputter-deposited Sn thin films and have been reported in other literature ${ }^{6,8}$ because $\mathrm{Sn}$ atoms have high adatom mobility and can easily form large clusters during preparation processes. For n-AS films deposited with substrate bias, the film morphologies are all similar to Fig. 3a, as shown in Fig. 3b and c, possibly because the n-AS film is already well-crystallized so that further crystallization is difficult. The AS sample is also shown in Fig. 3d with the same magnification for comparison.

For the AS samples, the surface is much more smooth and composed of nanosized primary particles $(5-10 \mathrm{~nm})$ only. This can be attributed to the reduced energy input during film growth, as discussed in the previous section.

Figure 4a-e shows the surface morphologies of AS thin films under different negative bias. The cluster sizes on the film surface increase as the bias increases (Fig. 4a-d). As the bias exceeds $70 \mathrm{~V}_{\text {DC }}$, much larger surface particles form (Fig. 4e), and the morphology is completely different from the lower biased ones. This confirms that the surface morphology was controlled by the energy input during film growth. The bias also induced an etching mechanism, which densifies the films by adatom relocation and deepening actions, and results in improved crystallization. Therefore, reducing the energy input during film growth by means of anode shielding can effectively produce nanocrystalline $\mathrm{Sn}$ thin films.

Crystallography.- Figure 5a and b shows the XRD patterns of AS and n-AS deposited Sn thin films with different substrate bias. The n-AS samples (as shown in Fig. 5a) exhibit typical Sn diffraction peaks based on the JCPDF cards. ${ }^{11}$ The intensities of diffraction peaks of these samples are much stronger than those of the AS samples. AS thin films show low-intensity diffraction peaks and exhibit near-amorphous features (Fig. 5b).

The crystallography (Figs. 5a and b) and surface morphology (Fig. 3 and 4) correspond well, where n-AS films form large surface particles and crystallized better than the AS films. The results can be
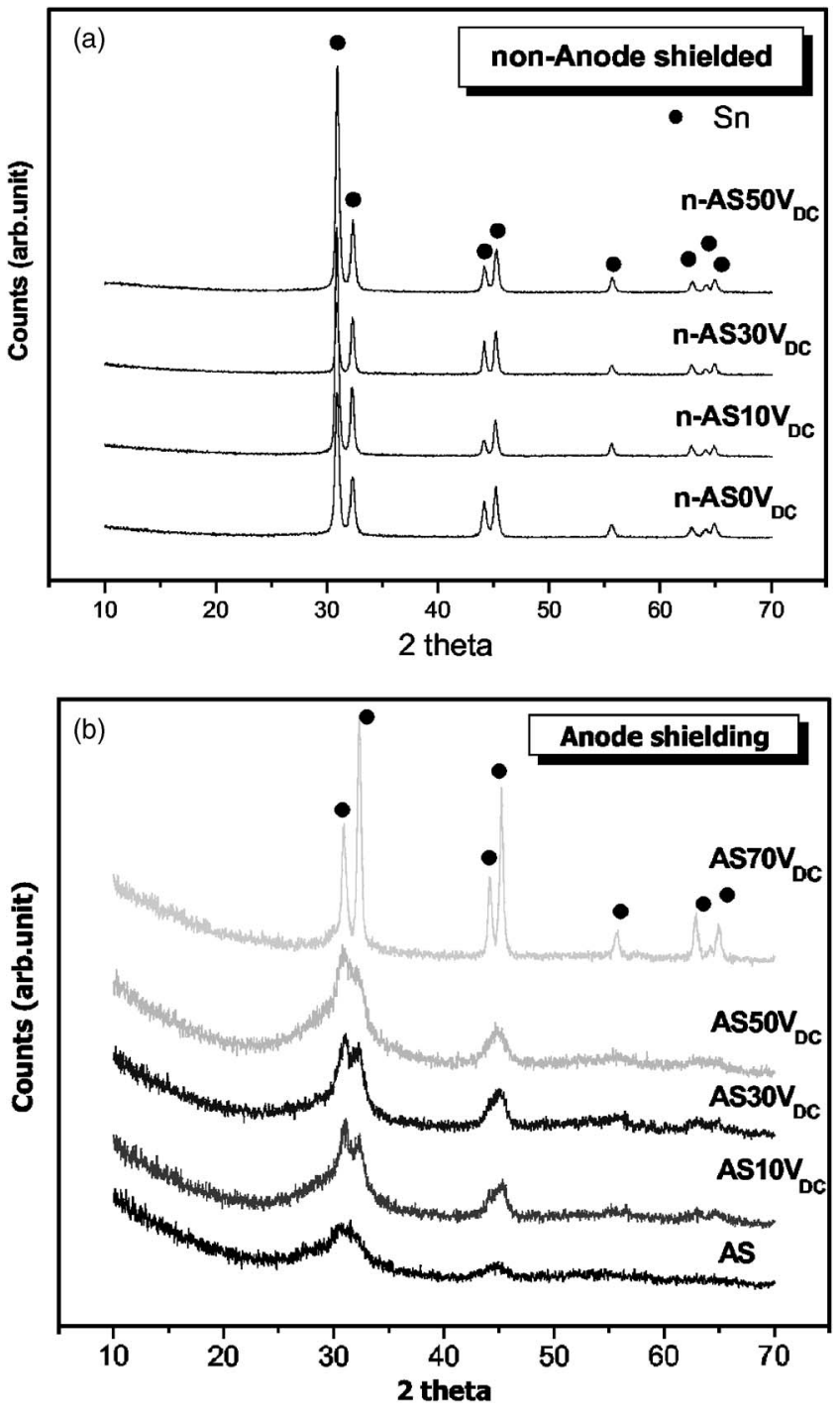

Figure 5. XRD patterns of (a) n-AS and (b) AS Sn thin films deposited under different bias.

attributed to the different plasma conditions during deposition. As discussed before, for films deposited without anode shielding the much larger ion flux irradiating on the growing films provides extra energy and an etching process which enhances the film crystallinity. However, further crystallization is not significant, as shown in Fig. $5 \mathrm{a}$, where the peak intensity and width are all similar as substrate bias increases.

The additional anode shielding confines the plasma and lowers the energy input during film growth, which provides an effective way to reduce the particle sizes of sputtered Sn thin films. For AS thin films deposited under different bias (Fig. 5b), it is observed that

\begin{tabular}{|c|c|c|c|c|c|c|}
\hline Samples & $\begin{array}{c}\text { Initial specific } \\
\text { capacity } \\
(\mathrm{mAh} / \mathrm{g})\end{array}$ & $\begin{array}{c}\text { Initial } \\
\text { efficiency } \\
(\%)\end{array}$ & $\begin{array}{c}\text { Initial } \\
\text { capacity } \\
(\mathrm{mAh} / \mathrm{g})\end{array}$ & $\begin{array}{c}\text { Capacity at } \\
\text { cycle } 20 \\
(\mathrm{mAh} / \mathrm{g})\end{array}$ & $\begin{array}{c}\text { Capacity at } \\
\text { cycle } 50 \\
(\mathrm{mAh} / \mathrm{g})\end{array}$ & $\begin{array}{c}\text { Capacity } \\
\text { retention } \\
\mathrm{C} 20 / \mathrm{C}^{\mathrm{a}}(\%)\end{array}$ \\
\hline AS & 963 & 86 & 744 & 522 & 432 & 70 \\
\hline n-AS & 868 & 90 & 783 & 205 & 179 & 26 \\
\hline $\mathrm{AS} 70 \mathrm{~V}_{\mathrm{DC}}$ & 864 & 51 & 446 & 374 & 281 & 43 \\
\hline
\end{tabular}

${ }^{a} \mathrm{C} 20 / \mathrm{C} 1$ ratio: Capacity at 20 cycle/capacity at 1 cycle $(\%)$. The uncertainty of the capacity is $\pm 5 \%$. 


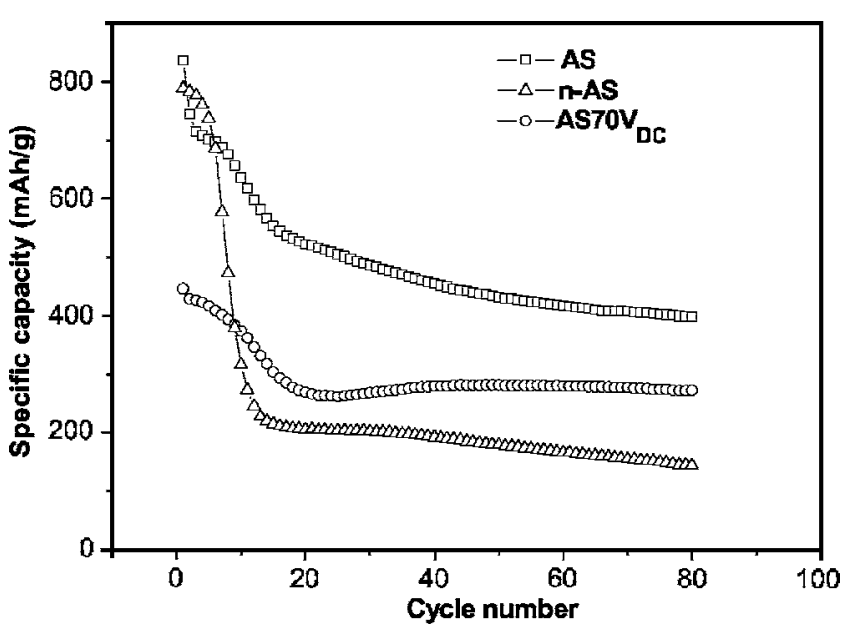

Figure 6. The electrochemical cycling test of Sn thin-film anodes, AS, n-AS, and $\mathrm{AS} 70 \mathrm{~V}_{\mathrm{DC}}$.

the AS Sn thin films exhibit near-amorphous structure at low substrate bias. As the substrate bias increases to $50 \mathrm{~V}_{\mathrm{DC}}$, the crystallinity of Sn thin films is gradually enhanced. At $70 \mathrm{~V}_{\mathrm{DC}}$, a well-crystallized Sn diffraction spectrum is recorded. It is supposed that the biasinduced ion bombardment during film growth provides an etching process and enough energy, as mentioned in the previous section, to enhance film crystallization during deposition.

Electrochemical tests. - Half-cells made of Sn thin films deposited in different conditions were tested. The electrochemical properties and cycle life performance of different thin films are detailed in Table II and shown in Fig. 6. The cells in Fig. 6 were cycled up to 80 cycles at $50 \mu \mathrm{A} / \mathrm{cm}^{2}$ for the charge step and $10 \mu \mathrm{A} / \mathrm{cm}^{2}$ for the discharge step between 2.50 and $0.05 \mathrm{~V}$. The larger charge current density prevents the occurrence of anomalous irreversible capacity at high voltage and avoids the decomposition of electrolyte. ${ }^{5}$

The initial and reversible capacities of all electrodes are higher than commercial carbonaceous anodes. The initial efficiencies are similar for the AS and n-AS samples. The AS70 $V_{D C}$ films shows lowest initial efficiency and reversible capacity, which may be due to plasma damage from ion energy ion bombardment. As the cycle number increases, the AS samples exhibit the highest capacity retention. After the first ten cycles, the reversible capacity of the n-AS samples decreases rapidly with increase of cycling number. The reversible capacity drops rapidly within 20 cycles to only $200 \mathrm{mAh} / \mathrm{g} \pm 5 \%$. Even the AS70 $\mathrm{V}_{\mathrm{DC}}$ samples perform better than the n-AS samples. The results may be attributed to the morphology of these samples. The n-AS samples with coarse and large particle sizes $(\sim \mu \mathrm{m})$ exhibit poor cycling ability. The AS70V $\mathrm{V}_{\mathrm{DC}}$ anodes, which also have large particle sizes $(\sim 100 \mathrm{~nm})$, show only a slightly higher reversible capacity $(281 \mathrm{mAh} / \mathrm{g} \pm 5 \%)$ after 50 cycles.

The rapid capacity fading in the first 15-20 cycles results from the formation of solid-electrolyte-interphase (SEI) layers, which are commonly observed for anodes used in $\mathrm{Li}$ and $\mathrm{Li}$-ion cells. In the reduction step, lithium reacts with electrolyte and anode materials, which produce a solid layer on the surface of the anode. Most of the reactions of SEI formation are irreversible or only partially reversible. In addition, for $\mathrm{Sn}$ anodes, the volume difference between $\mathrm{Li}_{x} \mathrm{Sn}$ alloy and $\mathrm{Sn}$ metal are large, resulting in significant volume changes during charge-discharge cycles. Large mechanical stresses result from the inhomogeneous expansion and large volume changes during lithium insertion/removal into/from the host matrix of $\mathrm{Sn}$ thin-film anodes. The stress induced by the large volume changes results in cracks, where the fresh Sn metal is exposed to electrolyte in the next cycles, and new SEI forms again. Therefore, the capacity fades during the first $15-20$ cycles. The reversible capacity stabilizes

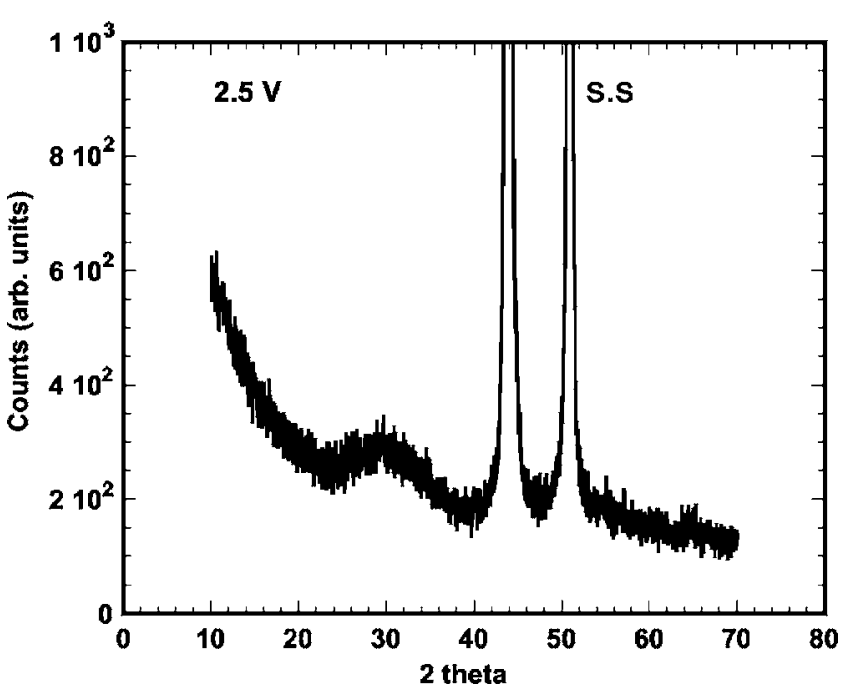

Figure 7. XRD patterns of the cycled AS Sn thin film at $2.5 \mathrm{~V}$. The substrate is stainless steel.

as the anode surface is well covered by the thick SEI layer, which prevents further exposure of fresh Sn metal to the electrolyte. The cracked particles lose the electric contact between each other or simply peel off. The well-crystallized n-AS and AS70V $V_{D C}$ films are not able to endure the large volume changes and suffer most from the cracking and crumbling, resulting in poor cycling properties.

AS samples show much better cycling ability. These samples maintain the capacity better in the cycling test and retain a capacity as high as $450 \mathrm{mAh} / \mathrm{g} \pm 5 \%$ up to 80 cycles, which means that the cycling ability of Sn electrodes can be improved by reducing the particle sizes. The smaller, nanocrystalline particles have larger, integral interparticle contact area per particle volume, which improves the adhesion over larger, well-crystallized particles. Figure 7 shows the XRD patterns of a postcycled AS film (at $2.5 \mathrm{~V}$ ). Only a nearamorphous background with sharp peaks of the stainless steel substrate can be observed.

With the setup of additional anode shielding, the technique developed here has demonstrated the ability to fabricate amorphous/ nanocrystalline Sn thin films with high reversible capacity.

\section{Conclusions}

Sn thin films with ultrasmall particle sizes were deposited using rf magnetron sputtering under anode shielding. It was demonstrated that additional anode shielding is an effective way to reduce the particle sizes. The influences of ion flux bombarding on the growing films are significant for the low-melting-temperature metal or alloy, such as Sn metal, and the reduction of ion flux by grounded anode shielding can effectively prevent agglomeration during film preparation, resulting in near-amorphous thin films composed of nanoscaled particles. The electrochemical properties of the deposited thin films were verified. The improvement in electrochemical properties can be mainly attributed to the increased interparticle contact area of the amorphous/nanocrystalline thin films. The cycling properties of the $\mathrm{Sn}$ thin-film anodes were significantly improved.

\section{Acknowledgments}

This research work was sponsored by Taiwan R.O.C. National Science Council under contract no. NSC 92-2216-E-035-024 and NSC 93-2216-E-035-024.

Feng Chia University assisted in meeting the publication costs of this article.

\section{References}

1. M. Winter and J. O. Besenhard, Electrochim. Acta, 45, 31 (1999).

2. J. Yang, M. Winter, and J. O. Besenhard, Solid State Ionics, 90, 281 (1996). 
3. R. A. Huggins, J. Power Sources, 81/82, 13 (1999).

4. L. Y. Beaulieu, S. D. Beattie, T. D. Hatchard, and J. R. Dahn, J. Electrochem. Soc., 150, A419 (2003).

5. S. D. Beattie, T. D. Hatchard, A. Bonakdarpour, K. C. Hewitt, and J. R. Dahn, J. Electrochem. Soc., 150, A701 (2003).

6. L. Y. Beaulieu, T. D. Hatchard, A. Bonakdarpour, M. D. Fleischauer, and J. R. Dahn, J. Electrochem. Soc., 150, A1457 (2003).

7. J. O. Besenhard, J. Yang, and M. Winter, J. Power Sources, 68, 87 (1997)

8. L. Y. Beaulieu, K. C. Hewitt, R. L. Turner, A. Bonakdarpour, A. A. Abdo, L.
Christensen, K. W. Eberman, L. J. Krause, and J. R. Dahn, J. Electrochem. Soc., 150, A149 (2003).

9. N. Tamura, R. Ohshita, M. Fujimoto, S. Fujitani, M. Kamino, and I. Yonezu, J. Power Sources, 107, 48 (2002).

10. K.-F. Chiu, M. G. Blamire, and Z. H. Barber, J. Vac. Sci. Technol. A, 17, 2891 (1999).

11. JCPDS card no. 04-0673, International Center for Diffraction Data, Swarthmore PA. 